\title{
Surgical Paddle Electrode Implantation as a Rescue Therapy to Failed Percutaneous Leads in Failed Back Surgery Syndrome Patients
}

\author{
Richard L. Witkam, BSc ${ }^{1,2}$; Marije L. Buijse, BSc ${ }^{1}$; Inge J.J. Arnts, MANP, PhD ${ }^{1}$; \\ Dylan J.H.A. Henssen, MD, PhD ${ }^{3}$; Kris C.P. Vissers, MD, PhD ${ }^{1}$; \\ Robert van Dongen, $M D, \mathrm{PhD}^{1}$; Erkan Kurt, $\mathrm{MD}^{2}$
}

\begin{abstract}
Introduction: Spinal cord stimulation (SCS) to treat failed back surgery syndrome (FBSS) can be provided with either percutaneously or surgically implanted electrodes. Percutaneous electrodes are considered the first choice in many pain practices, but surgical paddle electrodes can also be indicated if a percutaneous electrode fails to retain sufficient pain relief or if percutaneous implantation is considered unachievable. Although the current efficacy of surgical paddle electrodes has been based mainly upon pain intensity scores, the evidence on surgical paddle electrodes as a rescue to failed percutaneous electrodes remains even more scarce.

Objective: This study aimed to evaluate the safety and clinical effectiveness of rescue surgical paddle electrodes in FBSS patients, multidimensionally.

Materials and Methods: The occurrence of complications, pain intensity scores, psychosocial-related questionnaires, and medication intake were collected. Subsequently, a Quality-of-Life Index (QLI) was calculated. A clinically relevant effect was obtained if the minimal clinically important difference regarding pain intensity was reached.

Results: A total of 25 patients were included in the study. The pain intensity scores were significantly reduced ( $p<0.001)$, and clinically relevant reductions occurred during short-term ( $0-6$ months), mid-term ( $1-3$ years), and long-term follow-up ( $\geq 4$ years). The structural morphine usage and QLI were significantly decreased at short-term follow-up ( $p=0.038$ and $p=0.036$, respectively). Six complications occurred in five patients, of which, four concerned hardware-related problems and two were of biological origin.

Conclusion: SCS utilizing a surgical paddle electrode as a salvage treatment to failed conventional percutaneous cylindrical lead SCS can be practiced safely and effectively to treat FBSS. Because of potentially improved clinical effectiveness and cost-effectiveness resulting from fewer reoperations, a SCS treatment algorithm may benefit from expediting surgical paddle electrodes.
\end{abstract}

Keywords: Failed back surgery syndrome, long-term follow-up, revision surgery, spinal cord stimulation, surgical paddle electrode

Conflict of Interest: The authors reported no conflict of interest.

\section{INTRODUCTION}

Patients are diagnosed with failed back surgery syndrome (FBSS) if they report persistent or recurrent back pain or radicular leg pain after lumbosacral spine surgery that was technically and anatomically successful. ${ }^{1}$ Among other domains, FBSS patients tend to experience a lower quality of life (QoL) because of greater levels of pain. ${ }^{2}$ When previous conservative therapies fail to diminish pain sufficiently, spinal cord stimulation (SCS) can be provided because it has been shown to improve pain relief and QoL. ${ }^{3}$ In addition, SCS has been considered more effective than

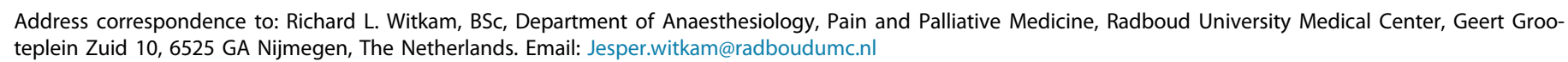

1 Department of Anaesthesiology, Pain and Palliative Medicine, Radboud University Medical Center, Nijmegen, The Netherlands;
2 Department of Neurosurgery, Radboud University Medical Center, Nijmegen, The Netherlands; and
3 Department of Medical Imaging, Radboud University Medical Center, Nijmegen, The Netherlands

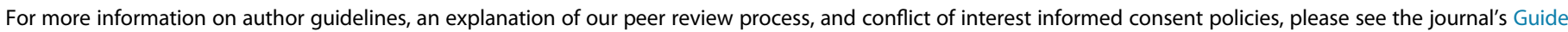
for Authors.

Source(s) of financial support: No funding was received for conducting this research. 
spinal reoperation, owing to a relatively high failure rate in terms of pain relief. 4

The SCS electrodes can be placed either percutaneously (ie, cylindrical electrodes) or surgically through a laminotomy or laminectomy (ie, surgical paddle electrode). ${ }^{5}$ Advantages of the surgical paddle electrode include its flatter shape with wider design, ${ }^{6}$ resulting in less distance between the lead contacts and the target dorsal columns. ${ }^{7}$ Because the electrical current preferably flows through the cerebrospinal fluid (CSF), the likelihood of selective dorsal column fiber stimulation is increased because the width of the dorsal CSF space is smaller. ${ }^{8}$ Subsequent technical advantages mentioned are superior paresthesia coverage ${ }^{9}$ and prolonged battery life or charge burden because of lower amplitude requirements. ${ }^{10}$ Also, because the posterior aspects of paddle electrodes are insulated, current can be directed toward the desired target and minimize the unwanted current spread, resulting in decreased battery load or even less or no uncomfortable side stimulations because of sensory fiber recruitment in the ligamentum flavum. ${ }^{11}$ Other technical differences lie within the "center-to-center spacing" (ie, the distance between adjacent electrode contacts), the higher number of contacts, and the presence of multiple columns of contacts on the surgical paddle electrode. ${ }^{12}$ Such variations in the electrode design are based on the concept of "stimulation selectivity,"13,14 implying that the maximum therapeutic benefit is achieved when the intended neuronal structures in the dorsal column are stimulated (ie, inhibition of the lateral spinothalamic tract and increased activity of the descending antinociceptive pathways), without undesired stimulation leading to unpleasant paresthesias. From a technical point of view, surgical paddle electrodes may be considered as a first-line or as an alternative treatment strategy. ${ }^{15}$ However, clinical evidence on direct comparison of the two types of electrodes remains scarce and mostly inconsistent. ${ }^{16,17}$ Although patients with a surgical paddle electrode were reported to be more likely to reduce analgesic intake, ${ }^{16}$ contradictory conclusions have been reported regarding complication rates because some favored percutaneous electrodes, ${ }^{9}$ whereas others showed that the lower reoperation rates of paddle electrodes become advantageous over time, especially from two years follow-up onwards. ${ }^{18}$ Although surgical implantation is more invasive, the incidences of spinal cord injury and spinal hematoma were shown to be fewer in a large cohort of patients. $^{19}$

Because implantation of surgical paddle electrodes is not available in every practice, the percutaneous cylindrical electrodes are more often considered first choice. When patients are first seen by anesthesiologists-pain physicians who perform the percutaneous trial of SCS, a percutaneous cylindrical lead system as a permanent implant is typically the first-choice treatment in these centers. Consequently, in these practice environments, most surgically inserted paddle electrodes are reserved for percutaneous electrode lead failure to retain sufficient pain relief or when any technical difficulties arise during percutaneous implantation. ${ }^{20}$ In these cases, surgical paddle electrodes are often implanted after failed percutaneous SCS treatments or when the percutaneous route is considered anatomically unachievable (eg, scar tissue in the dorsal epidural space, or scoliosis). Because there is a relative paucity of published information on the use of rescue surgical paddle implants for failed percutaneous cylindrical systems, our goal was to assess the safety and clinical outcomes multidimensionally, including pain intensity, psychosocial-related questionnaires and QoL, changes in medication intake, and complications in FBSS patients who received SCS surgical paddle implants as a rescue for percutaneous cylindrical systems. Also, the results of our study may increase awareness and support for the value of shared decision making within a multidisciplinary neuromodulation pain center that can provide both surgical paddle and percutaneous cylindrical implant options by actively engaging our neurosurgery colleagues throughout the treatment process.

\section{MATERIALS AND METHODS}

\section{Study Population}

Adult patients (aged $\geq 18$ years) having FBSS with an indication for SCS, treated with a surgical paddle electrode as a rescue after a failed percutaneous cylindrical system at our university medical center between March 2004 and December 2019, were retrospectively studied. All electrodes were implanted by the same neurosurgeon. Written informed consent was obtained for each patient. Ethical approval for conducting this study was granted by the medical research ethics committee (ie, CMO RadboudUMC; file number: 2020-6556).

\section{Data Retrieval}

An existing treatment data base was supplemented with retrospectively retrieved data from medical records for the current study. Data were collected from baseline (ie, before paddle electrode implantation) and follow-up (ie, three months, six months, one year, and each year consecutively). The main reason for surgical paddle electrode implantation as a revision to a previously implanted electrode was identified for each patient. Additionally, multiple technical and perioperative variables were obtained. Although the stimulation parameters may account for the clinical outcomes by surgical paddle electrodes, they were not collected because the current study's power was too low to analyze the influence of such parameters.

The primary outcome measures comprised the pain intensity scores by means of the visual analog scale (VAS) and complications associated with SCS therapy. Complications were classified into either hardware (eg, electrode migration) or biological (eg, infection), and whether surgical treatment was required. Secondary outcome measurements consisted of medication intake and multiple psychosocial-related questionnaires, including the Hospital Anxiety and Depression Scale (HADS), ${ }^{21}$ the Pain Catastrophizing Scale (PCS), ${ }^{22}$ the 36 -Item Short Form Survey (SF-36), ${ }^{23}$ and the McGill Pain Questionnaire Dutch Language Version (MPQ-DLV). ${ }^{24,25}$

\section{Statistical Assessment}

A clinically relevant effect was considered to be obtained if the minimal clinically important difference (MCID) regarding pain intensity (ie, at least 1.5 points reduction on the VAS scale) was reached. ${ }^{26}$ A Quality-of-Life Index (QLI) was determined based on nine questions of the MPQ-DLV (range: 0-27 points; the lower the score, the better the QoL). In addition, the medication intake was quantified according to the Medication Quantification Scale (MQS), ${ }^{27}$ and the used opioid dosage was converted into a total morphine equivalent dose by means of standard conversion tables. Subsequently, the total daily morphine intake was split into a structural dose and a dose of occasional use.

To test for statistical significance between baseline and followup, the paired sample $t$-test was carried out. All statistic tests were two-sided, and $p<0.05$ was considered statistically 
significant. All analyses were performed with the use of SPSS (version 25.0; IBM Corp, Armonk, NY).

\section{RESULTS}

A total of 25 patients who received SCS therapy through surgically implanted paddle electrodes were included in this study (Table 1). The study cohort comprised 14 men and 11 women. The median age at implantation was 50.3 years (38.8-68.8 years). The implantable pulse generator was implanted in either the left gluteal region ( $n=17$ ) or the left abdominal wall $(n=8)$. The median postoperative follow-up was 43 months (3-194 months). Follow-up moments were divided into three periods: short-term (0-six months), mid-term (1-3 years), and long-term ( $\geq 4$ years follow-up, maximum of 16 years in one patient).
Twenty-three electrodes were implanted under general anesthetics without intraoperative monitoring. Paresthesia testing under spinal anesthesia was performed in the other two patients. For all patients, the primary intended surgical approach was a single-level unilateral interlaminar approach under microscopic magnification, at the same level of which the previous percutaneous electrode was placed if the patient's pain distribution was unchanged. This standard surgical approach was sufficient in only eight patients. If it was impossible to position the electrode in the midline (eg, because of epidural fibrosis or scar tissue), which was confirmed on an anterior-posterior view radiography, the approach was extended to the contralateral side (ie, single-level bilateral interlaminar approach) $(n=7)$. In case the lead could not be progressed to the adjacent intended level, a bilateral, multilevel interlaminar approach was performed $(n=10)$. Laterally orientated radiography was used to confirm a flat position of the electrode.

Table 1. Patient Characteristics $(N=25)$

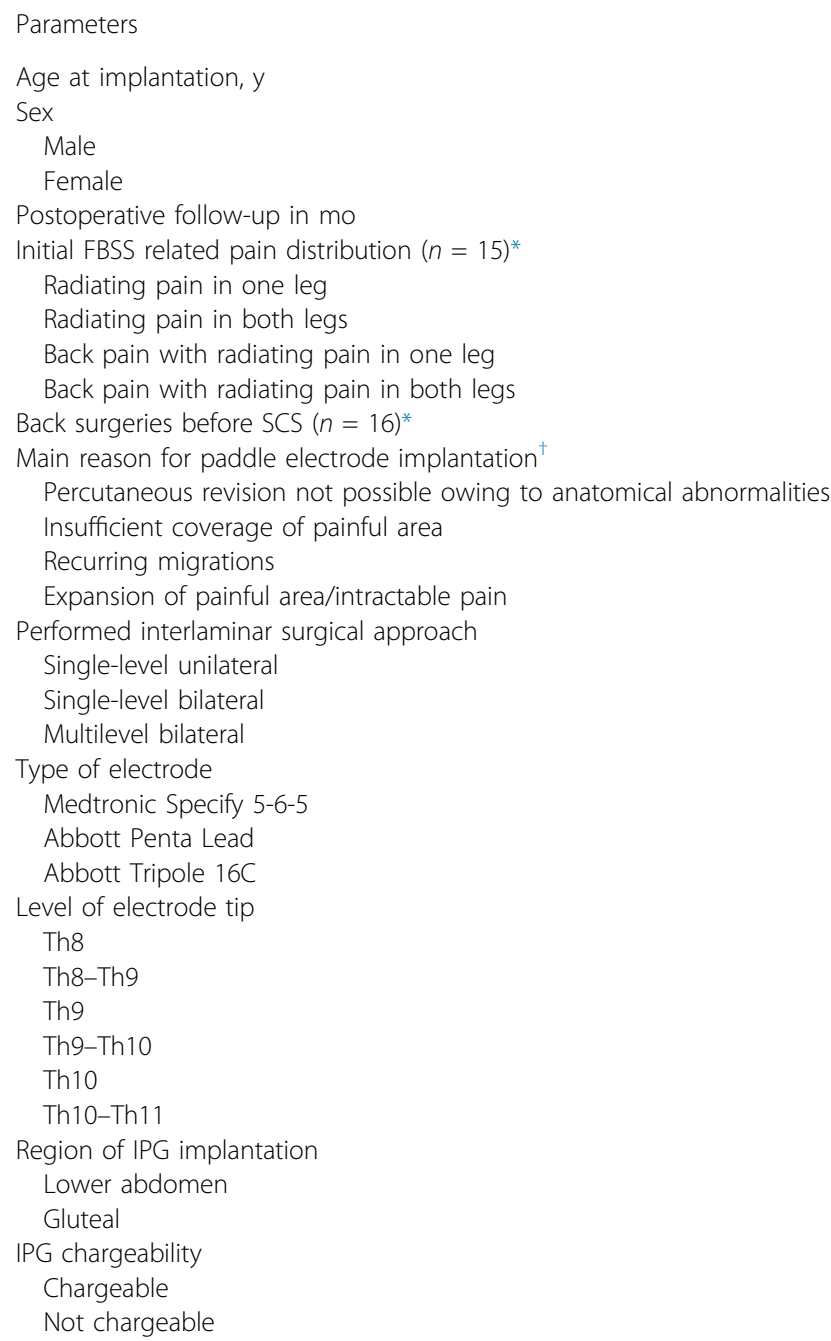

nitial FBSS related pain distribution $(n=15)^{*}$

Value

$50.3(38.8-68.8)$

$14(56.0 \%)$

$11(44.0 \%)$

$43.0(3.0-194.0)$

$2(8.0 \%)$

$2(8.0 \%)$

$3(12.0 \%)$

$8(32.0 \%)$

$2.0(1.0-6.0)$

$10(40.0 \%)$

8 (32.0\%)

4 (16.0\%)

3 (12.0\%)

8 (32.0\%)

7 (28.0\%)

$10(40.0 \%)$

20 (80.0\%)

$3(12.0 \%)$

$2(8.0 \%)$

3 (12.0\%)

7 (28.0\%)

5 (20.0\%)

6 (24.0\%)

1 (4.0\%)

3 (12.0\%)

8 (32.0\%)

$17(68.0 \%)$

5 (20.0\%)

20 (80.0\%)

The results in the right column are either depicted as their median and range or as their frequency of occurrence

IPG, implantable pulse generator.

*Data were not retrievable for some patients because of being lost to follow-up.

${ }^{\dagger}$ Although multiple reasons for paddle electrode implantation were applicable for some patients, the main reason was determined. 
Overall, a bilateral approach was required in most of the patients ( $n=17 ; 68.0 \%$ ), indicating the added complexity of reimplantation. Although direct relationships between this difficulty and explanatory variables could not be established based on our results, we observed more epidural fibrosis in patients who previously received multiple percutaneous leads, especially when one of those leads became infected. Hypertrophy of the flavum ligament also impeded surgical paddle placement in some patients. It must also be noted that in some patients with unilateral pain, a slight deviation of the electrode to the ipsilateral side was accepted. Otherwise, these patients would have required a bilateral or multilevel approach instead of a unilateral approach. Finally, before anchoring the surgical paddle electrode, its contact points were audited through impedance measures.

Pain intensity scores, medication intake, and questionnaire data were not reported in all patients within each follow-up period. Therefore, the number of patients and their total number of observations are depicted for each measurement at every followup period (Tables 2 and 3). The main reasons for these missing data were extended follow-up intervals over time, as well as some patients who chose to visit outpatient clinics closer to their homes.

\section{Pain Intensity}

The baseline, mean preoperative VAS score of all patients was $7.36 \pm 1.48$. Pain intensity scores were statistically significantly reduced for all three follow-up periods (Table 2). Regarding shortterm follow-up, the average VAS was $5.05 \pm 2.04(p<0.001)$. Particularly, 23 of 25 patients reported a decreased VAS score. However, both patients who disclosed an increase reported a decreased VAS score at longer follow-up. Concerning the mid- and long-term followup, the average pain intensity scores were $5.00 \pm 1.94(p<0.001)$ and $4.94 \pm 1.85(p<0.001)$, respectively. In terms of clinical relevance, the MCID was observed in $>50.0 \%$ of the patients for all three follow-up periods (ie, $59.1 \%, 71.4 \%$, and $86.7 \%$, respectively).

\section{Medication Intake}

The three most frequently used medication classes were benzodiazepines, antidepressants, and anticonvulsants. Because the amount of medication intake is positively correlated to the MQS, a reduced MQS score indicates less medication intake. Within the current study, the average MQS scores did not decrease significantly at any follow-up period (ie, short-term: $p=0.065$; mid-term: $p=0.391$; long-term: $p=0.195$ ) (Table 3). In contrast, the amount of structural morphine usage was statistically significantly reduced by $17.93 \mathrm{mg}$ per day on average at short-term follow-up $(p=0.038)$. However, regarding midand long-term follow-up, the decrease was reported to be $1.56 \mathrm{mg}$ ( $p=0.831)$ and $6.57 \mathrm{mg}(p=0.492)$, respectively. The amount of morphine being taken as occasional analgesics was reported not to be significantly reduced at any follow-up period.

\section{Psychosocial-related Questionnaires}

Neither the HADS subscores nor the HADS total score showed statistically significant differences (Table 3). Regarding the PCS questionnaire, the total score and helplessness score were significantly reduced at short-term follow-up $(p=0.029$ and $p=0.028$, respectively). The PCS total score remained to be statistically significant ( $p=0.028$ ) reduced at mid-term, but not at long-term follow-up ( $p=0.298$ ). Rumination was found to be statistically significantly improved at mid-term follow-up $(p=0.034)$. The only score of the SF-36 questionnaire that was found to be statistically significantly improved was the general health score at short-term follow-up $(p=0.030)$.

\section{Quality of Life}

The QLI, derived from the MPQ-DLV questionnaire, showed to be statistically significant improved at short-term $(p=0.036)$, but not at mid-term ( $p=0.542)$ and long-term $(p=0.333)$ follow-up (Table 3).

\section{Complication Rates}

Six complications occurred in five patients, of which four required surgical intervention (Table 4). Four of six complications concerned hardware problems, including two electrode fractures or malfunctions and two device components causing painful sensations. The other two complications were of biological origin and comprised two infections. Although both required intravenous antibiotics, one was also treated through surgical intervention (ie, complete removal of the SCS system). In the latter case, reimplantation was performed at least three months beyond the removal of the initial SCS system.

\section{DISCUSSION}

Within the current study, in which surgical paddle electrodes as a rescue therapy to percutaneous leads were studied, pain intensity score decreases were statistically significant and clinically relevant at the short-term (0-6 months), mid-term (1-3 years), and longterm follow-up ( $\geq 4$ years). One reason for these promising results could be the low reoperation rate because no electrode migrations occurred. The structural morphine usage was statistically significantly reduced at short-term follow-up. Despite the enhanced complexity of the outlined rescue surgical paddles, only two infections (8.0\%) were noticed. A similar increase in complexity of implant and increased infection rates were also reported in a similar subpopulation of patients. ${ }^{20}$

Two patients reported a short-term increase in pain owing to other painful comorbidities (ie, cervical disc herniation and gout), improving after adequate treatment. Therefore, it could be debated
Table 2. Pain Intensity Scores (VAS) Before and After SCS Therapy Using a Rescue Surgical Paddle Electrode.

Baseline $\quad$ 0-6 mo follow-up 1-3 y follow-up
VAS Mean $=7.36, \mathrm{SD}=1.48 \quad$ Mean $=5.05, \mathrm{SD}=2.04 \quad p<0.001^{*} \quad$ Mean $=5.00, \mathrm{SD}=1.94 \quad p<0.001^{*} \quad$ Mean $=4.94, \mathrm{SD}=1.85 \quad p<0.001^{*}$ $N=25 \quad N=22, x=39 \quad N=21, x=47 \quad N=15, x=42$
For each follow-up period, the number of included patients is depicted as " $N$," together with their overall number of observations in the corresponding period as "x." Because not all data during follow-up were retrievable for each patient, the numbers of patients and observations differ across short-, mid-, and long-term follow-up.
*Statistically significant differences.




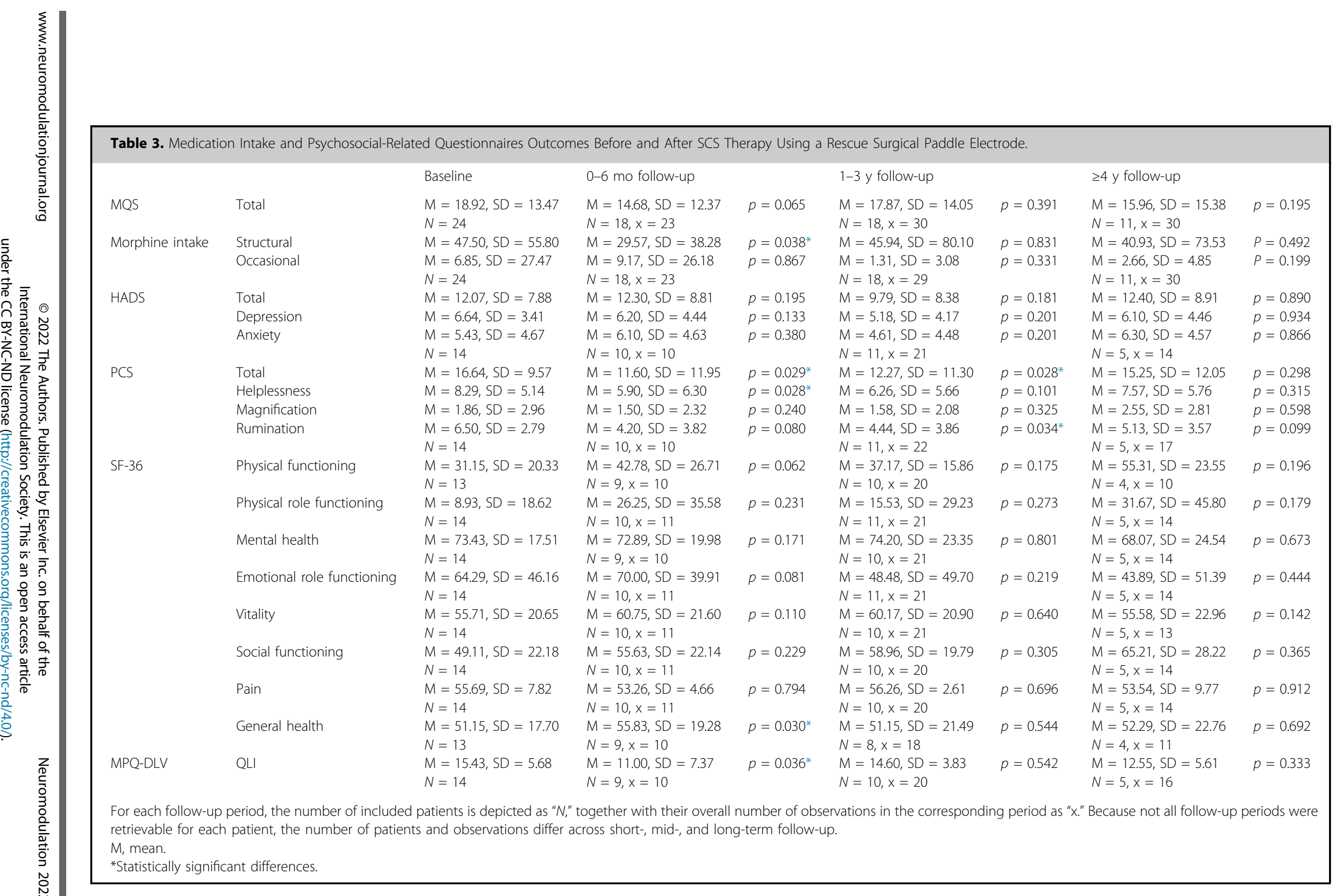


Table 4. Complications Associated With SCS Therapy Using Rescue Surgical Paddle Electrodes.

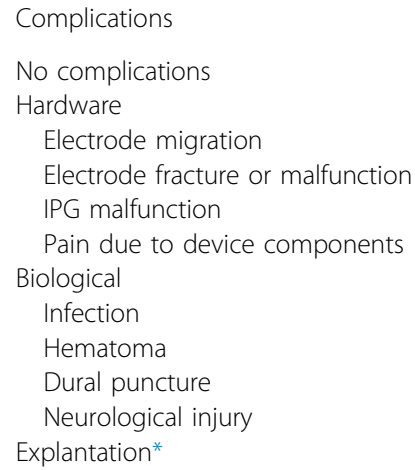

One patient endured both electrode malfunctioning on some contact points and mechanical pain at the inferior border of the IPG, which was in the left gluteal region. However, the electrode was not revised because optimal pain relief was not obtained before its malfunctioning, and the perioperative risk was considered too high because of comorbidities.

that all our patients responded to SCS therapy in terms of pain relief. However, our results would still have been dissimilar to those of Villavicencio et $\mathrm{al}^{17}$ who reported that all 12 surgical paddle electrode patients experienced $\geq 50.0 \%$ pain reduction. This dissimilarity could be a coincidence with such low numbers of inclusions. However, it may also be declared by the fact that patients who had their percutaneous electrode revised were also included within the current study because such patients tend to respond worse to a revisionary surgical paddle electrode treatment. ${ }^{20}$ Additionally, the same study showed a patient-reported satisfaction rate of $62.5 \%$ at 33 months of follow-up, which appeared to result from significant pain reduction and sufficient coverage of the painful area. ${ }^{20}$ Hence, SCS using paddle electrodes seems clinically relevant in diminishing FBSS related pain, also after multiple percutaneous implantations.

To conclude from our cohort, a low reoperation rate might be considered a positive outcome of using surgical paddle electrodes, especially regarding long-term follow-up. Because surgical paddles are less likely to migrate, they could provide more adequate pain relief. $^{28}$ Previously, Cameron ${ }^{29}$ described a percutaneous electrode migration rate of $13.2 \%(n=2753)$, whereas percutaneously implanted paddle electrodes migrated in $2.5 \%,{ }^{30}$ and the incidence of a surgical paddle electrode migration was merely $1.1 \%$ among 509 patients. ${ }^{5}$ During our median follow-up of 43 months (range: 3-194 months), no surgical revisions owing to an electrode migration were required. This could be the result of a more robust anchoring technique combined with a minimally surgical approach, during which nothing of the lamina was removed. However, the best anchoring method has not been determined, ${ }^{31}$ and Pahapill ${ }^{32}$ disclosed zero revisions required by a lead migration in a large cohort of implants without anchoring. Overall, perhaps independent of the place and type of anchoring, surgical paddle leads appear to contribute to clinical effectiveness and cost-effectiveness ${ }^{18}$ because they require fewer reoperations, even at long-term follow-up, when they are utilized as a rescue therapy in difficult implantations.

Regarding structural morphine usage, a statistically significant reduction was found at short-term follow-up. However, North et $\mathrm{al}^{16}$ also reported a significantly reduced analgesic intake at 2.9 years follow-up, favoring surgical paddle electrodes over percutaneous electrodes. The EMPOWER trial by St Jude Medical, in which 149 patients with a penta electrode were analyzed (37.2\%), disclosed a partial diminishment or completely reduced intake (33\%) concerningopioids or narcotics at three months follow-up. ${ }^{33}$ Another prospective investigation in both percutaneous as paddle electrodes showed that 32 of $53(60.4 \%)$ patients completely stopped their medication intake at one-year follow-up. ${ }^{34}$ Although the MCID was reached in all subcohorts, the mean reduction in VAS was most prominent in patients who had never used opioids previously. Subsequently, the authors suggested that preoperative elimination of opioid intake might enhance clinical outcomes. ${ }^{34}$ To elaborate, preoperative opioid intake could also be seen as a negative predictor, either because of a more intractable pain syndrome or because of a patient who is less able to cope with the current situation. Fewer side effects because of a lowered medication intake would enhance QoL-related outcomes, which could be disparate to a reduced pain intensity score, even if that might be the only measured outcome. ${ }^{35}$ Therefore, a multifaceted outcome parameter including medication intake and pain intensity scores would be beneficial for properly assessing clinical outcomes.

The presented infection rate $(8.0 \%)$, including one superficial infection which did not require surgical intervention, lies within the previously published incidence range of $2.5 \%$ to $14.0 \%$ by Bendersky et al. ${ }^{28} \mathrm{~A}$ negative contributor to our infection rate may be that few surgical paddle electrode implantations have been performed over many years. Besides, academic centers are more likely to report higher infection rates, possibly resulting from the enhanced complexity of their cases. ${ }^{36}$ However, a large academic center recently reported a $0.0 \%$ infection rate for surgical paddle implants. $^{37}$ Also, reimplantation procedures are believed to increase infection rates. $^{20,37}$ For example, extensive amounts of epidural fibrosis in the current study population impeded the surgical implantation and lengthened the surgical procedure as a result, which increases the infection risk. For example, each previous percutaneous implantation is believed to lengthen the subsequent percutaneous implantation by 15 minutes. ${ }^{38}$ It could be hypothesized that the presented infection rates would have been lower without the presence of such unfavorable conditions. Despite the invasive character of surgical paddle electrode 
implantation, no difference was found regarding the infection rate between the surgical paddle and percutaneous electrodes in 2628 patients with different pathologies. ${ }^{36}$ The reported infection rates coincided with earlier research, because a difference was found at neither 30 days nor 90 days of follow-up $(n=9072) .{ }^{18}$ For surgical paddle electrodes specifically, Lee et $\mathrm{al}^{39}$ reported no infections after trialed implantation in 12 patients. Another study, in which 16 of 22 trialed patients were permanently implanted, also reported no infections. ${ }^{40}$ Recent reports in both academic and nonacademic settings have illustrated the safety of surgical paddle electrodes with near-zero electrode migrations or fracture rates and no infections. $^{32,37}$ Therefore, it could be hypothesized that surgical paddle electrode placement is a safe therapy and is also a salvage treatment for failing percutaneous electrodes. Finally, the implementation and perhaps further optimization of an infection care bundle approach may suppress infection rates even further across neuromodulation centers, leading to reduced morbidity and enhanced cost-effectiveness. ${ }^{37}$

Although our study is only the second reporting on the outcomes of rescue paddle implants for failed percutaneous permanent cylindrical systems, ${ }^{20}$ the current study was limited by a smaller population size and data being lost to follow-up because of its retrospective nature. Some patients were referred to our neuromodulation center to receive a surgical paddle electrode, whereas data on the percutaneous implantation remained at the initial implantation site. Though, the current study presents data on rescue paddle electrodes in a homogenous cohort. Furthermore, it contributes to building a larger amount of clinical experience regarding SCS therapy through a surgical paddle electrode after previously failed percutaneous cylindrical electrode treatment. Although not generalizable at this point, at least for our center, the current results indicated that earlier consideration of implanting a surgical paddle electrode might be beneficial. Optimally, an (inter)national registry could be created to demonstrate the clinical utility (eg, effectiveness and long-term safety) through extensive data sets.

\section{CONCLUSION}

SCS using a surgical paddle electrode as a rescue to failed conventional percutaneous cylindrical lead SCS treatment can be practiced safely and effectively to treat FBSS, even in patients with difficult implantations owing to unfavorable conditions such as extensive epidural fibrosis or scarring. It may be hypothesized that within a multidisciplinary SCS therapy algorithm, outcomes might be improved upon expediting a full discussion regarding surgical paddle electrodes, if clinically appropriate and feasible for both the patient and neuromodulation center. Virgin surgical paddle electrode implantations could improve clinical effectiveness and cost-effectiveness over the long term because fewer reoperations may be required. Nevertheless, robust comparative studies are needed, specifically for tailoring each patient's needs through a holistic approach. Multifaceted outcome parameters, including medication intake and psychosocial-related questionnaires, should be utilized to better understand the clinical utility this implantation technique provides.

\section{Authorship Statements}

Richard L. Witkam, Marije L. Buijse, and Erkan Kurt designed and conducted the study, including data collection. Inge J.J. Arnts aided in patient recruitment and data collection. The data analysis and interpretation were performed by all authors throughout multiple interdisciplinary sessions. Richard L. Witkam prepared the manuscript, with indispensable intellectual input from all coauthors. All authors approved the final manuscript.

\section{How to Cite This Article}

Witkam R.L., Buijse M.L., Arnts I.J.J., Henssen D.J.H.A., Vissers K.C.P., van Dongen R., Kurt E. 2022. Surgical Paddle Electrode Implantation as a Rescue Therapy to Failed Percutaneous Leads in Failed Back Surgery Syndrome Patients.

Neuromodulation 2022; 25: 745-752.

\section{REFERENCES}

1. Thomson S. Failed back surgery syndrome-definition, epidemiology and demographics. Br J Pain. 2013;7:56-59.

2. Thomson S, Jacques L. Demographic characteristics of patients with severe neuropathic pain secondary to failed back surgery syndrome. Pain Pract. 2009:9:206-215.

3. Grider JS, Manchikanti L, Carayannopoulos A, et al. Effectiveness of spinal cord stimulation in chronic spinal pain: a systematic review. Pain Phys. 2016;19:E33-E54.

4. North RB, Kidd DH, Farrokhi F, Piantadosi SA. Spinal cord stimulation versus repeated lumbosacral spine surgery for chronic pain: a randomized, controlled trial. Neurosurgery. 2005;56:98-106 [discussion: 106-107].

5. Barolat G. Experience with 509 plate electrodes implanted epidurally from $\mathrm{C} 1$ to L1. Stereotact Funct Neurosurg. 1993:61:60-79.

6. Oakley JC. Spinal cord stimulation: patient selection, technique, and outcomes Neurosurg Clin N Am. 2003;14:365-380, vi.

7. Son $\mathrm{BC}, \mathrm{Ko} H C$, Choi JG. Compression of thoracic spinal cord with decreased cerebrospinal fluid space after implantation of paddle lead spinal cord stimulation at T9: a three-dimensional myelographic computed tomography study. World Neurosurg. 2018;118:e323-e334.

8. Holsheimer J. Effectiveness of spinal cord stimulation in the management of chronic pain: analysis of technical drawbacks and solutions. Neurosurgery. 1997;40:990-996 [discussions: 996-999].

9. Rosenow JM, Stanton-Hicks M, Rezai AR, Henderson JM. Failure modes of spinal cord stimulation hardware. J Neurosurg Spine. 2006;5:183-190.

10. North RB, Kidd DH, Olin JC, Sieracki JM. Spinal cord stimulation electrode design: prospective, randomized, controlled trial comparing percutaneous and laminectomy electrodes-part I: technical outcomes. Neurosurgery. 2002;51:381-389 [discussion: 389-390].

11. North RB, Lanning A, Hessels R, Cutchis PN. Spinal cord stimulation with percu taneous and plate electrodes: side effects and quantitative comparisons. Neurosurg Focus. 1997;2:e3.

12. Bradley K. The technology: the anatomy of a spinal cord and nerve root stimulator: the lead and the power source. Pain Med. 2006:7:S27-S34.

13. Holsheimer J, Wesselink WA. Effect of anode-cathode configuration on paresthesia coverage in spinal cord stimulation. Neurosurgery. 1997:41:654-659 [discussion: 659-660].

14. Holsheimer J, Struijk JJ, Tas NR. Effects of electrode geometry and combination on nerve fibre selectivity in spinal cord stimulation. Med Biol Eng Comput. 1995:33:676-682.

15. Manola L, Holsheimer J. Technical performance of percutaneous and laminectomy leads analyzed by modeling. Neuromodulation. 2004;7:231-241.

16. North RB, Kidd DH, Petrucci L, Dorsi MJ. Spinal cord stimulation electrode design: a prospective, randomized, controlled trial comparing percutaneous with laminectomy electrodes: part II-clinical outcomes. Neurosurgery. 2005;57:990-996 [discussion: 990-996].

17. Villavicencio AT, Leveque JC, Rubin L, Bulsara K, Gorecki JP. Laminectomy versus percutaneous electrode placement for spinal cord stimulation. Neurosurgery. 2000;46:399-405 [discussion: 405-406].

18. Babu R, Hazzard MA, Huang KT, et al. Outcomes of percutaneous and paddle lead implantation for spinal cord stimulation: a comparative analysis of complications, reoperation rates, and health-care costs. Neuromodulation. 2013;16:418-426 [discussion: 426-427].

19. Petraglia 3rd FW, Farber SH, Gramer R, et al. The incidence of spinal cord injury in implantation of percutaneous and paddle electrodes for spinal cord stimulation. Neuromodulation. 2016;19:85-90. 
20. Matias CM, Amit A, Lempka SF, et al. Long-term outcomes after replacement of percutaneous leads with paddle leads in a retrospective cohort of patients with spinal cord stimulation systems. Neurosurgery. 2014;75:430-436 [discussion: 436].

21. Spinhoven $P$, Ormel J, Sloekers PP, Kempen GI, Speckens AE, Van Hemert AM A validation study of the Hospital Anxiety and Depression Scale (HADS) in different groups of Dutch subjects. Psychol Med. 1997;27:363-370.

22. Sullivan MJL, Bishop SR, Pivik J. The Pain Catastrophizing Scale: development and validation. Psychol Assess. 1995;7:524-532.

23. Aaronson NK, Muller M, Cohen PD, et al. Translation, validation, and norming of the Dutch language version of the SF-36 Health Survey in community and chronic disease populations. J Clin Epidemiol. 1998;51:1055-1068.

24. Vanderiet $\mathrm{K}$, Adriaensen $\mathrm{H}$, Carton $\mathrm{H}$, Vertommen $\mathrm{H}$. The McGill Pain Questionnaire constructed for the Dutch language (MPQ-DV). Preliminary data concerning reliability and validity. Pain. 1987:30:395-408.

25. Verkes RJ, Vanderiet K, Vertommen H, Van der Kloot W, Meij J. De MPQ-DLV: een standaard Nederlandstalige versie van de McGill Pain Questionnaire voor België en Nederland. Swets \& Zeitlinger; 1989:57-78.

26. Ostelo RW, Deyo RA, Stratford P, et al. Interpreting change scores for pain and functional status in low back pain: towards international consensus regarding minimal important change. Spine (Phila Pa 1976). 2008:33:90-94.

27. Harden RN, Weinland SR, Remble TA, et al. Medication Quantification Scale Version III: update in medication classes and revised detriment weights by survey of American Pain Society Physicians. J Pain. 2005;6:364-371.

28. Bendersky D, Yampolsky C. Is spinal cord stimulation safe? A review of its complications. World Neurosurg. 2014;82:1359-1368.

29. Cameron T. Safety and efficacy of spinal cord stimulation for the treatment of chronic pain: a 20-year literature review. J Neurosurg. 2004;100(3 suppl Spine):254267.

30. Kinfe TM, Schu S, Quack FJ, Wille C, Vesper J. Percutaneous implanted paddle lead for spinal cord stimulation: technical considerations and long-term follow-up. Neuromodulation. 2012:15:402-407.

31. Levy R, Henderson J, Slavin K, et al. Incidence and avoidance of neurologic complications with paddle type spinal cord stimulation leads. Neuromodulation. 2011:14:412-422 [discussion: 422].

32. Pahapill PA. Incidence of revision surgery in a large cohort of patients with thoracic surgical three-column paddle leads: a retrospective case review. Neuromodulation. 2015:18:367-375

33. Rosenberg J, Fabi A, Candido K, et al. Spinal cord stimulation provides pain relief with improved psychosocial function: results from EMP3OWER. Pain Med. 2016:17:2311-2325.

34. Gee L, Smith HC, Ghulam-Jelani Z, et al. Spinal cord stimulation for the treatment of chronic pain reduces opioid use and results in superior clinical outcomes when used without opioids. Neurosurgery. 2019;84:217-226.

35. Witkam RL, Kurt E, van Dongen R, et al. Experiences from the patient perspective on spinal cord stimulation for failed back surgery syndrome: a qualitatively driven mixed method analysis. Neuromodulation. 2021;24:112-125.

36. Hoelzer BC, Bendel MA, Deer TR, et al. Spinal cord stimulator implant infection rates and risk factors: a multicenter retrospective study. Neuromodulation. 2017;20:558-562

37. Arocho-Quinones EV, Huang CC, Ward BD, Pahapill PA. Care bundle approach to minimizing infection rates after neurosurgical implants for neuromodulation: a single-surgeon experience. World Neurosurg. 2019;128:e87-e97.

38. Kin $\mathrm{K}$, Agari $\mathrm{T}$, Yasuhara $\mathrm{T}$, et al. The factors affecting the difficulty of percutaneous cylindrical electrode placement for spinal cord stimulation. World Neurosurg 2018;113:e391-e398.

39. Lee JJ, Simpson RK, Dalm B. Permanent paddle-lead trial for spinal cord stimulation. Cureus. 2018:10:e2645.
40. Pahapill PA. Surgical paddle-lead placement for screening trials of spinal cord stimulation. Neuromodulation. 2014;17:346-348 [discussion: 348]

\section{COMMENT}

The authors clearly and concisely describe their experience in providing surgical paddle implants as a rescue option for failed back surgery patients with failed percutaneous leads. In addition to VAS pain scores, they also provide long-term outcomes in this challenging subpopulation of patients in an extensive multidimensional fashion. There remains a paucity of such data; thus, this report, although small and retrospective, adds to our knowledge in this arena. In addition to reminding us of the potential advantages of surgical paddle vs percutaneous cylindrical lead implants, they also provide compelling evidence that it would be in the best interests of our patients that the option of a surgical vs percutaneous implant should be discussed in a multidisciplinary, comprehensive SCS neuromodulation environment and presented to patients who passed their initial trial of SCS. These include 1) the greater challenge of implanting a paddle lead owing to adhesions and scarring from failed percutaneous leads ( $40 \%$ need for multilevel bilateral exposures in this study), and 2) the need for additional procedures that are associated with greater complications such as infections. These can all lead to less-thanoptimal outcomes, cost-effectiveness, and perhaps even future limitation of patient access to these therapies. I would have liked to know 1) how the outcomes of these rescue paddle systems compared with the outcomes of the failed percutaneous systems before they had failed, and 2) what the failure rate was for their percutaneously implanted cylindrical lead systems compared with failure rates of their paddle systems that were initially implanted up front. Although not clearly shown in the literature, anecdotal evidence suggests that the greater delay between successful trial percutaneous cylindrical lead pull and surgical paddle lead implant, the greater trial lead associated adhesions/scar development with subsequent increased probability for the need for an extended exposure/dissection of the epidural space for optimal paddle lead placement. Personally, we have seen this, especially when two magnetic resonance imaging safe trial leads are placed adjacent to each other from all vendors.

Ahmed Awad, MD; Peter Pahapill, MD, PhD Milwaukee, WI, USA 